\title{
Composite nature of hadrons and Bose-Einstein correlations
}

\author{
A.Bialas ${ }^{1, a}$ \\ ${ }^{1}$ M.Smoluchowski Institute of Physics, Jagellonian University, , ul.Lojasiewicza 11, 30-348 Krakow, Poland
}

\begin{abstract}
I am reporting results of two papers, written together with W.Florkowski and K.Zalewski [1,2], discussing the consequences of the observation [3] that, due to their composite nature and thus finite size, hadrons observed in the HBT measurements must be correlated in space-time. Using the blast-wave model [4] adjusted [1] to ALICE data on the measured HBT radii in $p p$ collisions at $7 \mathrm{TeV}$ [5], the full Bose-Einstein correlation functions in three direction (out, side, long) are evaluated. The results are presented together with some additional comments.
\end{abstract}

\section{Introduction}

Interpretation of the measurements of Bose-Einstein correlation function is usually discussed using the formula

$$
C\left(P_{12}, Q\right) \equiv \frac{N\left(p_{1}, p_{2}\right)}{N\left(p_{1}\right) N\left(p_{2}\right)}=1+\frac{\left|\int d x_{1} d x_{2} e^{i Q x} w\left(P_{12}, x\right)\right|^{2}}{\int d x_{1} w\left(p_{1}, x_{1}\right) \int d x_{2} w\left(p_{2}, x_{2}\right)} \equiv C_{u}\left(P_{12}, Q\right) \geq 1
$$

where $p_{1}, x_{1}$ and $p_{2}, x_{2}$ are momenta and positions of particles, $w(p, x)$ is their corresponding distribution (Wigner function). $N\left(p_{1}, p_{2}\right)$ is the measured number of particle pairs, $N(p)$ is the measured single particle distribution, and

$$
P_{12}=\left(p_{1}+p_{2}\right) / 2 ; \quad Q=p_{1}-p_{2},
$$

Several experiments have shown, however, that this formula does not describe correctly the existing data [6-8] because the inequality on the R.H.S. of (1) is not satisfied.

The explanation of this problem can be found by observing that (1) is only an approximation to the general formula $[9,10]$

$$
C\left(P_{12}, Q\right)=1+\frac{\int d x_{1} d x_{2} e^{i Q\left(x_{1}-x_{2}\right)} W\left(P_{12}, P_{12} ; x_{1}, x_{2}\right)}{\int d x_{1} w\left(p_{1}, x_{1}\right) \int d x_{2} w\left(p_{2}, x_{2}\right)}
$$

where $W\left(P_{12}, P_{12} ; x_{1}, x_{2}\right)$ is the distribution in momentum and in space-time (Wigner function) of the two particles. Eq. (1) is obtained from (3) under the assumption that the produced particles are uncorrelated, i.e.

$$
W\left(p_{1}, p_{2} ; x_{1}, x_{2}\right)=w\left(p_{1}, x_{1}\right) w\left(p_{2}, x_{2}\right)
$$

\footnotetext{
ae-mail: bialas@th.if.uj.edu.pl
} 


\section{MIXING OF QUARKS}
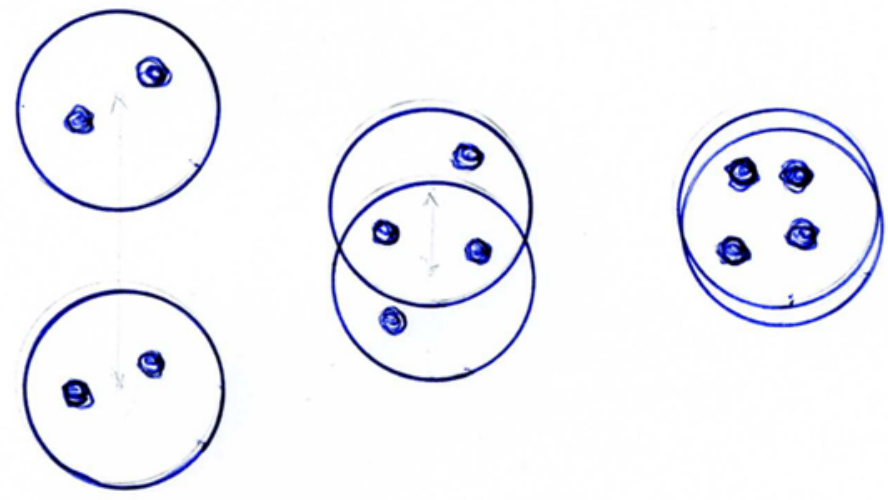

Figure 1. Schematic illustration of the mixing of the constituents of two hadrons

Thus violation of the inequality (1) implies that the produced particles must be correlated.

It was suggested in [3] that such correlations in space-time must be necessarily present because observed hadrons are not point-like particles but rather systems composed of a certain number of more elementary constituents. Consequently, when two hadrons happen to occur at a distance smaller than their actual size, their constituents mix, their wave functions are distorted and the quantum interference cannot take place. In other words, the HBT experiment cannot see hadrons which happen to be too close to each other. The idea is illustrated schematically in Fig 1.

To take this effect into account, we propose to replace (4) by

$$
W\left(p_{1}, p_{2} ; x_{1}, x_{2}\right)=w\left(p_{1}, x_{1}\right) w\left(p_{2}, x_{2}\right)\left[1-D\left(x_{1}-x_{2}\right)\right]
$$

where the "cut-off" function $D\left(x_{1}-x_{2}\right)$ equals 1 at $x_{1}=x_{2}$ and vanishes at distances larger than, say, $1 \mathrm{fm}$.

Introducing (5) into (3) one obtains for the correlation function

$$
C\left(P_{12}, Q\right)=C_{u}\left(P_{12}, Q\right)-C_{D}\left(P_{12}, Q\right)
$$

where $C_{u}\left(P_{12}, Q\right)$ is given by (1) and

$$
C_{D}\left(P_{12}, Q\right)=\frac{\int d x_{1} d x_{2} e^{i Q\left(x_{1}-x_{2}\right)} w\left(P_{12}, x_{1}\right) w\left(P_{12}, x_{2}\right) D\left(x_{1}-x_{2}\right)}{\int d x_{1} w\left(p_{1}, x_{1}\right) \int d x_{2} w\left(p_{2}, x_{2}\right)}
$$

One sees from (6) that the inequality (1) is no longer compulsory. 


\section{Qualitative picture}

It is essential to realise that the two terms on the R.H.S. of (6) differ drastically in their $Q$-dependence. Indeed, in the first one the integral takes contributions from the full space-time volume where hadrons are produced and, consequently, the dependence on $Q$ is rather sharp, reflecting the size of the system. On the other hand, in the second term the integration volume is strongly restricted by the cut-off function $D\left(x_{1}-x_{2}\right)$ and thus the dependence on $Q$ is much weaker. Therefore, although the second term gives only a small correction at $Q \approx 0$, it may dominate at large $Q$, where the data show that the inequality (1) is violated.

This observation allows to formulate the approximate procedure to estimate the magnitude of the effects we are discussing:

STEP ONE: Choose a model describing the considered process and fix its parameters by adjusting to data at small $Q$, ignoring the correction from the space-time correlation.

STEP TWO: Introduce the cut-off function and evaluate the full correlation function in the wide region of $Q$.

STEP THREE: verify that the description at small $Q$ is not destroyed.

Then the results of STEP TWO represent a prediction of the model which may be compared with data at large $Q$ in order to find a proper form of the cut-off function $D\left(x_{1}-x_{2}\right)$

\section{Blast-wave model}

To realise this program, we employed the blast-wave model $[4,9,11]$. (its details are described in [1]). The parameters were adjusted to account for the HBT radii measured by the ALICE collaboration in $p p$ collisions at $7 \mathrm{TeV}$ [5] (STEP ONE). Then the HBT correlation functions were evaluated in the region up to $Q=1 \mathrm{GeV}$ (STEP TWO), using the cut-off function in the Gaussian form

$$
D\left(x_{1}-x_{2}\right)=e^{-l^{2} / \Delta^{2}}
$$

where $l$ is the space-time distance (along the hyperbola $\tau=\sqrt{t^{2}-z^{2}}=$ const), and $\Delta=1 \mathrm{fm}$. It was checked that introducing the cut-off function does not change the HBT radii (STEP THREE).

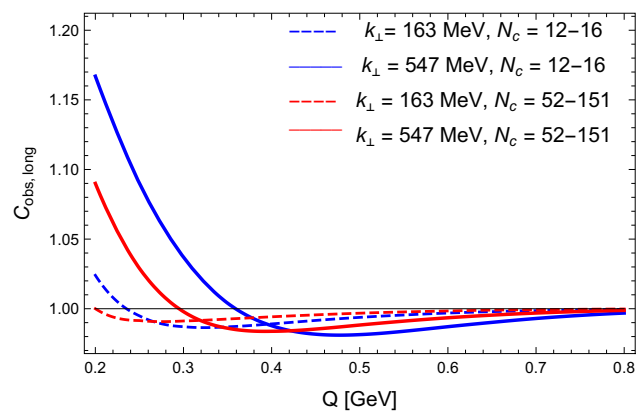

Figure 2. (Color online) Correlation function $C_{\mathrm{obs}}$ for the long direction in the interval $0.2 \mathrm{GeV} \leq Q \leq 0.8 \mathrm{GeV}$ (normalised to 1 at $\mathrm{Q}=1 \mathrm{GeV}$ ). The dashed lines describe the results for $k_{\perp}=163 \mathrm{MeV}$ and the two multiplicity classes: $N_{c}=12-16$ and $N_{c}=52-151$. The solid lines describe the results for $k_{\perp}=547 \mathrm{MeV}$ and the same two multiplicity classes. 


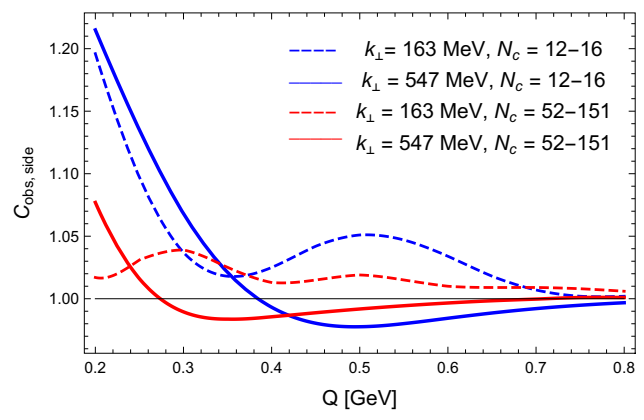

Figure 3. The same as Fig. 2 but for the side direction.

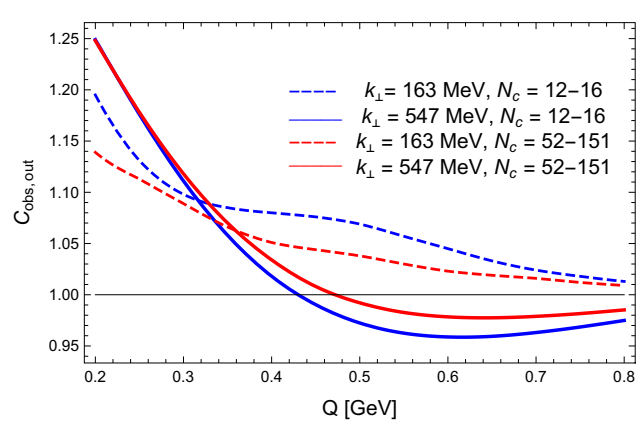

Figure 4. The same as Fig. 2 but for the out direction.

It turns out that the results (published in [2]) depend substantially on (i) the direction in which the measurement is performed, (ii) the transverse momentum of the pair and (iii) the multiplicity of the collision. They are shown in Figs. 2- 4 (for a detailed description, see [2]). We also found that the magnitude of the corrections decreases with decreasing ratio of the cut-off parameter $\Delta$ to the total size of the system. Thus one expects that the effects shall be less visible in collisions involving nuclei.

Unfortunately, there is no substantial sensitivity to the shape of the cut-off function: Calculations with the sharp cut-off at the distance $\Delta \approx 0.75 \mathrm{fm}$ gave very similar results.

\section{Summary and comments}

To summarize, we estimated the effects of the space-time correlations, implied by the composite nature of the produced hadrons, on the HBT measurements. Our calculations indicate that the precise measurements of the HBT correlation functions in the region of $Q$ from $200 \mathrm{MeV}$ up to $1 \mathrm{GeV}$ may reveal a rich structure, depending nontrivially on the direction of the measurement and on other characteristics of the produced hadronic system. Detailed comparison of data with the model calculations may, eventually, pin down the value of the cut-off parameter (and thus also the density) at which the produced hadrons melt into the quark-gluon plasma. Although rather difficult, it seems that such measurements are worth to undertake. 


\section{Acknowledgements}

I would like to thank my collaborators for help in preparing this report. Thanks are also due to Adam Kisiel for introduction into data from the ALICE experiment. This investigation was supported by the grant from the National Science Centre (Narodowe Centrum Nauki) DEC-2013/09/B/ST2/00497.

\section{References}

[1] A. Bialas, W. Florkowski and K. Zalewski, J. Phys. G: Nucl.Part.Phys. 42 (2015) 045001.

[2] A. Bialas, W. Florkowski and K. Zalewski, : Phys. Letters B748, 9 (2015)

[3] A. Bialas and K. Zalewski, Phys. Lett. B727, 182 (2013)

[4] F. Retiere and M. A. Lisa, Phys.Rev. C70, 044907 (2004).

[5] K. Aamodt et al. [ALICE Collaboration], Phys. Rev. D 84, 112004 (2011).

[6] M. Acchiari et al. [L3 coll.] Phys. Lett. B458 (1999) 517; P. Achard et al. [L3 coll.], Eur. Phys.J. C71 (2011) 1648.

[7] G. Abbiendi et al. [OPAL coll.], Eur. Phys. J. C16 (2000) 423; P. Abreu et al. [DELPHI coll.] Phys. Lett. B471 (2000) 460; A. Heister et al. [ALEPH coll.] Eur. Phys. J. C36 (2004) 460.

[8] V. Khachatryan et al. [CMS coll.], JEHP 1105 (2011) 029; L. Perroni, PoS (WPCF2011) 015.

[9] For a review, see W. Florkowski, "Phenomenology of Ultra-Relativistic Heavy-Ion Collisions", (Singapore, World Scientific, 2010); M. A. Lisa, S. Pratt, R. Soltz and U. Wiedemann, Ann. Rev. Nucl. Part. Sci. 55, 357 (2005).

[10] For an elementary derivation, see, e.g. A. Bialas and A. Krzywicki, Phys. Lett. B 354, 134 (1995).

[11] A. Bialas, W. Florkowski and K. Zalewski, Acta Phys. Pol. B45 (2014) 1883. 